\title{
Neutrophil to lymphocyte ratio and Platelet to lymphocyte ratio: Novel markers of inflammation in Psoriasis
}

\author{
Shakti Kumar Yadav ${ }^{1}$, Santosh Sharma ${ }^{1}$, Sompal Singh ${ }^{1}$, V.K. Khurana ${ }^{2}$ and Namrata Sarin ${ }^{1 *}$ \\ 'Department of Pathology, North Delhi Municipal Corporation Medical College and Hindu Rao Hospital, Delhi, India. \\ ${ }^{2}$ Department of Dermatology, North Delhi Municipal Corporation Medical College and Hindu Rao Hospital, Delhi, India.
}

\section{ABSTRACT}

Background: Psoriasis is a systemic, autoimmune chronic inflammatory disease. Novel inflammatory biomarkers like neutrophil-tolymphocyte ratio (NLR) and platelet-to-lymphocyte ratio (PLR) may help in evaluation of systemic inflammation. Aim of the present study was to evaluate NLR and PLR in patients of psoriasis and to correlate them with psoriasis area severity index (PASI) score.

Methods: The study was done in a tertiary care hospital. A study was performed with 40 psoriasis patients (study group) and 20 healthy control subjects. Complete hemogram was determined using the 5-part hematology analyser. NLR and PLR values were calculated from the appropriate absolute cell counts. Mann-Whitney U test was applied to compare the data between patients and controls. Pearson correlation was used to find correlation between NLR, PLR and PASI.

Result: Mean PLR was 121.79 (42.36) for study group and 101.42 (SD 52.28) for the control group. We found that PLR was significantly higher in study group than the control group ( $\mathrm{p}$ value $=0.041$ ). There was a positive correlation between PASI and PLR ( $\mathrm{r}=0.476, \mathrm{p}$ value 0.002). We found no difference in NLR values between patients and controls as well as no correlation between PASI and NLR in the study group.

Conclusion: PLR can be used as a biomarker to measure and monitor the disease activity in psoriasis patients at the time of diagnosis and follow up. This can also aid in preventing comorbidities in psoriasis patients.

\section{Keywords: Neutrophil-to-lymphocyte Ratio, Platelet-to-lymphocyte Ratio, Psoriasis, PASI}

\section{Introduction}

Psoriasis is a systemic, autoimmune chronic inflammatory disease. The worldwide prevalence of psoriasis ranges from 2 to $3 \%$ which may vary depending upon the environmental and genetic factors. ${ }^{[1]}$ It can occur at any age although, majority of the patients present in third and fourth decade of life. ${ }^{[2]}$

The noticeable immunopathological mechanism is the prominent infiltration of the skin by inflammatory cells. ${ }^{[3]}$ These inflammatory cells are not only detected in tissues but also in the peripheral blood of the psoriasis patients.

${ }^{[4]}$ Defining disease severity in psoriasis is important to plan suitable, safe and effective treatment. Hence, many authors have tried to identify useful biomarkers in psoriasis patients' peripheral blood, which can be used to assess the disease severity. Though, evaluation of psoriasis severity is difficult and there is no single validated tool that can evaluate all the aspects of the severity of psoriasis..$^{[5]}$

The commonly used clinical scale for evaluation of disease severity is Psoriasis Area Severity Index (PASI) which is based in area of body and degree of skin scaling. However, its applications are limited due variation among clinicians.
Various authors have evaluated disease activity in psoriasis by measuring serum levels of cytokines, cell adhesion molecules and C-reactive protein in peripheral blood. ${ }^{[6,7]}$ However, intricate laboratory setup and qualified personnel are needed to perform these test, which along with high costs limit their routine use.

NLR and PLR are recently recognized as a simple and potential biomarker of systemic inflammation in various diseases including myocardial infarction, atherosclerosis, hypertension, metabolic syndrome, diabetes mellitus, ulcerative colitis, end stage renal disease and rheumatoid arthritis. ${ }^{[8-12]}$ However, only a few studies have evaluated the role of NLR and/or PLR in psoriasis. ${ }^{[4,13,14]}$

Polat et al. ${ }^{[13]}$ has evaluated role of NLR and PLR in psoriasis in Turkish patients. They found that NLR and PLR were significantly elevated in psoriasis patients. They also found positive correlation of NLR and PLR with PASI. However, no author has evaluated the role of NLR and PLR in psoriasis in Indian subcontinent.

In this study, we have evaluated the NLR and PLR in psoriasis patients and healthy control subjects. In addition, we also tried to evaluate their correlation with disease severity using PASI. 


\section{Materials and Methods}

A total of 60 individuals were enrolled for the study. The two groups included 40 psoriasis patients (cases) and 20 matched healthy control. Sample size estimation was done using the Z-test method for difference of mean, based on previous similar study, ${ }^{[13]}$ taking statistical power of 80 . Approvals for the study was taken from the hospital scientific and ethical committee. Informed consent was taken from subjects of both groups. Age, sex and other demographic details were noted. Patients diagnosed with malignant tumours and other systemic inflammatory diseases were excluded from the study. Venous blood samples from both cases and control subjects were collected in a $3 \mathrm{ml} \mathrm{K}_{2}$ EDTA vacuum tube. Complete blood counts for both sample types were analysed in a 5 part haematology analyser Sysmex XT2000i (Sysmex Corporation, Japan). Absolute neutrophil and absolute lymphocyte count were determined.

NLR was calculated by dividing absolute neutrophil count by absolute lymphocyte count. PLR was obtained by dividing platelet count by absolute lymphocyte count. The PASI (psoriasis area severity index) score was calculated using the formula. ${ }^{[15]}$

PASI $=0.1(\mathrm{EH}+\mathrm{IH}+\mathrm{DH}) \mathrm{AH}+0.2(\mathrm{EU}+\mathrm{IU}+\mathrm{D}=) \mathrm{AU}$ $+0.3(\mathrm{ET}+\mathrm{IT}+\mathrm{DT}) \mathrm{AT}+0.4(\mathrm{EL}+\mathrm{IL}+\mathrm{AL}) \mathrm{AL}$

E-Erythema, I-Indurations, D-Desquamation, A-Area of skin affected, H-Head, U- Upper limb, T-Trunk, L-Lower limb. The area of skin affected was taken as 0 (no skin involvement), 1 (1-9\% skin affected), 2 (10-29\%), 3 (30$49 \%), 4(50-69 \%), 5(70-89 \%)$ and 6 (90-100\%). PASI Score value below 10 was defined as mild, 10-20 as moderate and more than 20 as severe psoriasis

Statistical Analysis: Continuous variables were expressed as mean \pm standard deviation. Mann-Whitney U test was used to compare NLR and PLR between cases and controls. Pearson's correlation was used to find correlation between NLR, PLR and PASI. A p-value of $<0.05$ was considered as significant.

\section{Result}

The mean age of the study group was 36 (SD 18) years. Males were $26(65 \%)$ and females were $14(35 \%)$. In our study 22 (55\%) patient had mild psoriasis, $11(26.2 \%)$ had moderate psoriasis and $7(17.5 \%)$ had severe psoriasis.
Mean PLR was 121.79 (SD 42.36) for study group and 101.42 (SD 52.28) for the control group (figure-1). We found that PLR was significantly higher in study group than the control group ( $\mathrm{p}$ value $=0.041)$. There was a positive correlation between PASI and PLR $(r=0.476$, $p$ value 0.002) (figure-2).

We compared NLR and PLR in cases and controls. The mean NLR of study group was 2.29 (SD 1.29) while control group was 1.82 (SD 0.73) (figure-3). However, there was no difference in NLR values between the two groups (figure-4). We also found no correlation between PASI and NLR in the study group.

We found that PLR was highest in patients with severe psoriasis. Comparison between NLR and PLR scores of mild, moderate and severe psoriasis are given in table- 1 .

\section{Discussion}

Disease activity in psoriasis is a multifactorial process and presently there is no universal marker to assess disease activity and systemic inflammation. Role of various cytokines is studied by some authors as biomarker for psoriasis, although these are not cost effective and required elaborate laboratory set up. ${ }^{[13]}$

NLR and PLR are recently recognized as a potential biomarker of systemic inflammation in various diseases, like myocardial infarction, atherosclerosis, hypertension, metabolic syndrome and diabetes mellitus. ${ }^{[8-10]}$

We did not find any difference of NLR between psoriasis patients and control group and there was no correlation between PASI and NLR in the study group. Similar to our study, Ataseven et al. ${ }^{[16]}$ found no correlation between PASI and NLR, however, contrary to our results, they showed higher levels of NLR in study group $(2.19 \pm 1.11)$ than the control group $(1.80 \pm 0.72$, p value 0.011$)$. Yurtdas et al. ${ }^{[17]}$ in their study about NLR and subclinical atherosclerosis in psoriasis patients found that NLR levels were higher in study group as compared to the control group $(p=0.005)$. This difference between the results should be confirmed in a study with larger sample.

Very few authors have studied the role of PLR in psoriasis. In our study, we found that the PLR levels were significantly higher in psoriasis patients as compared to the control group and PLR showed a positive correlation with

Table 1: Comparison of mean NLR and PLR score in mild, moderate and severe psoriasis.

\begin{tabular}{|c|c|c|}
\hline Psoriasis severity (PASI) & NLR (SD) & PLR (SD) \\
\hline Mild (<10) & $2.14(1.21)$ & $112.24(41.97)$ \\
\hline Moderate (10-20) & $2.66(1.33)$ & $117.35(28.67)$ \\
\hline Severe (>20) & $2.16(1.51)$ & $158.82(46.45)$ \\
\hline
\end{tabular}




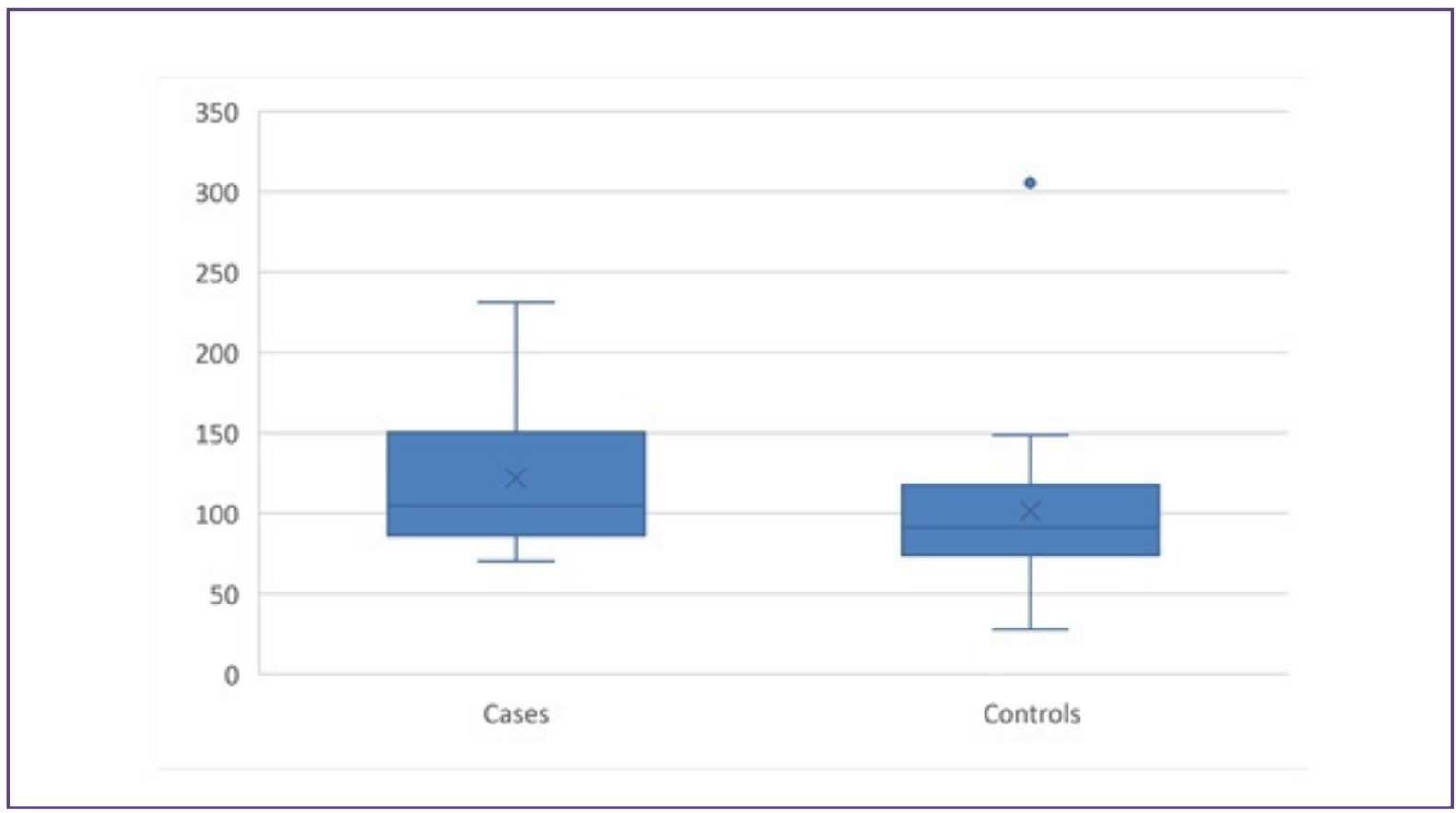

Fig. 1: Mean PLR in cases and controls.

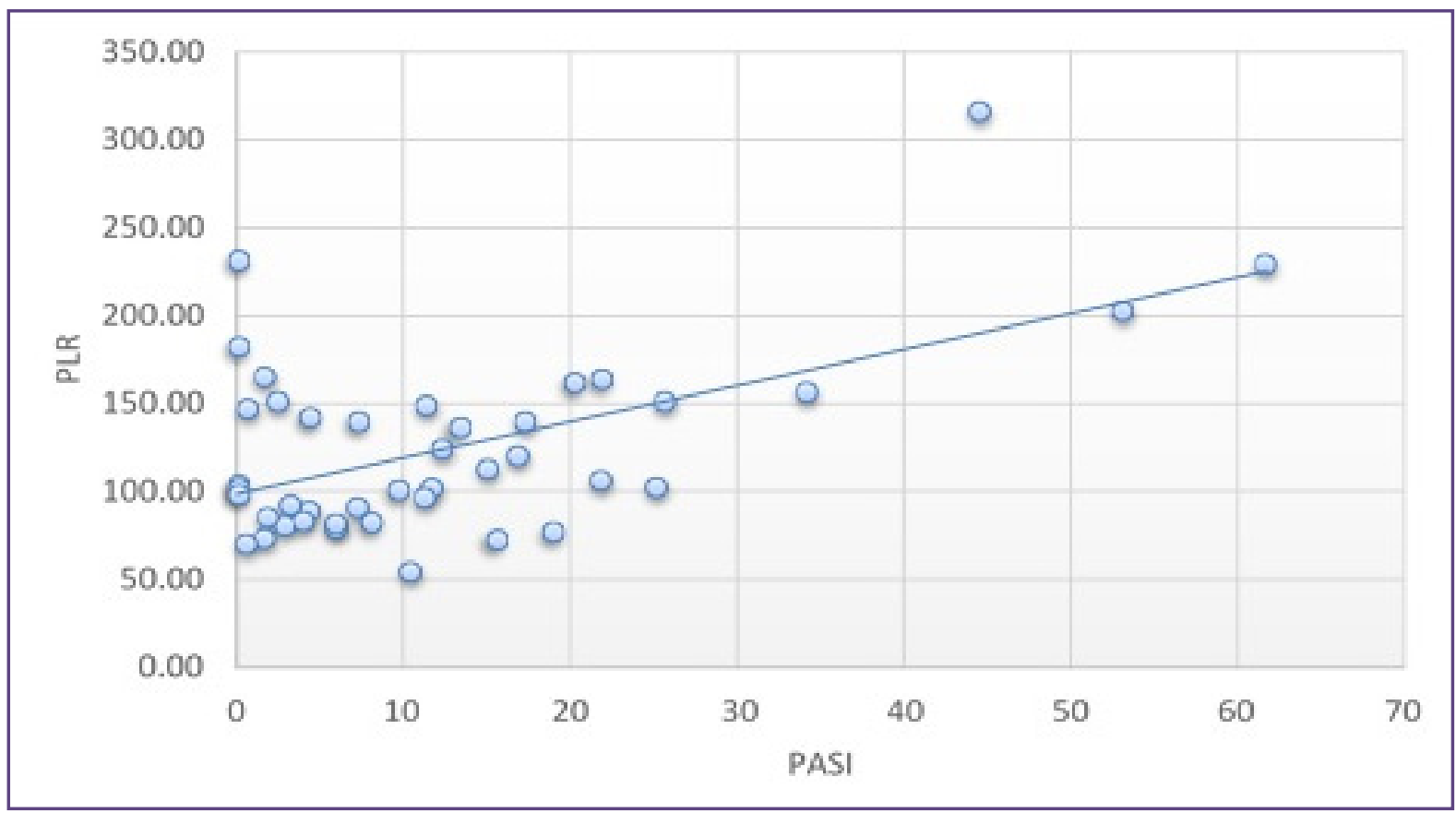

Fig. 2: Correlation between PASI and PLR $(r=0.560$, $p$ value $<0.001)$. 


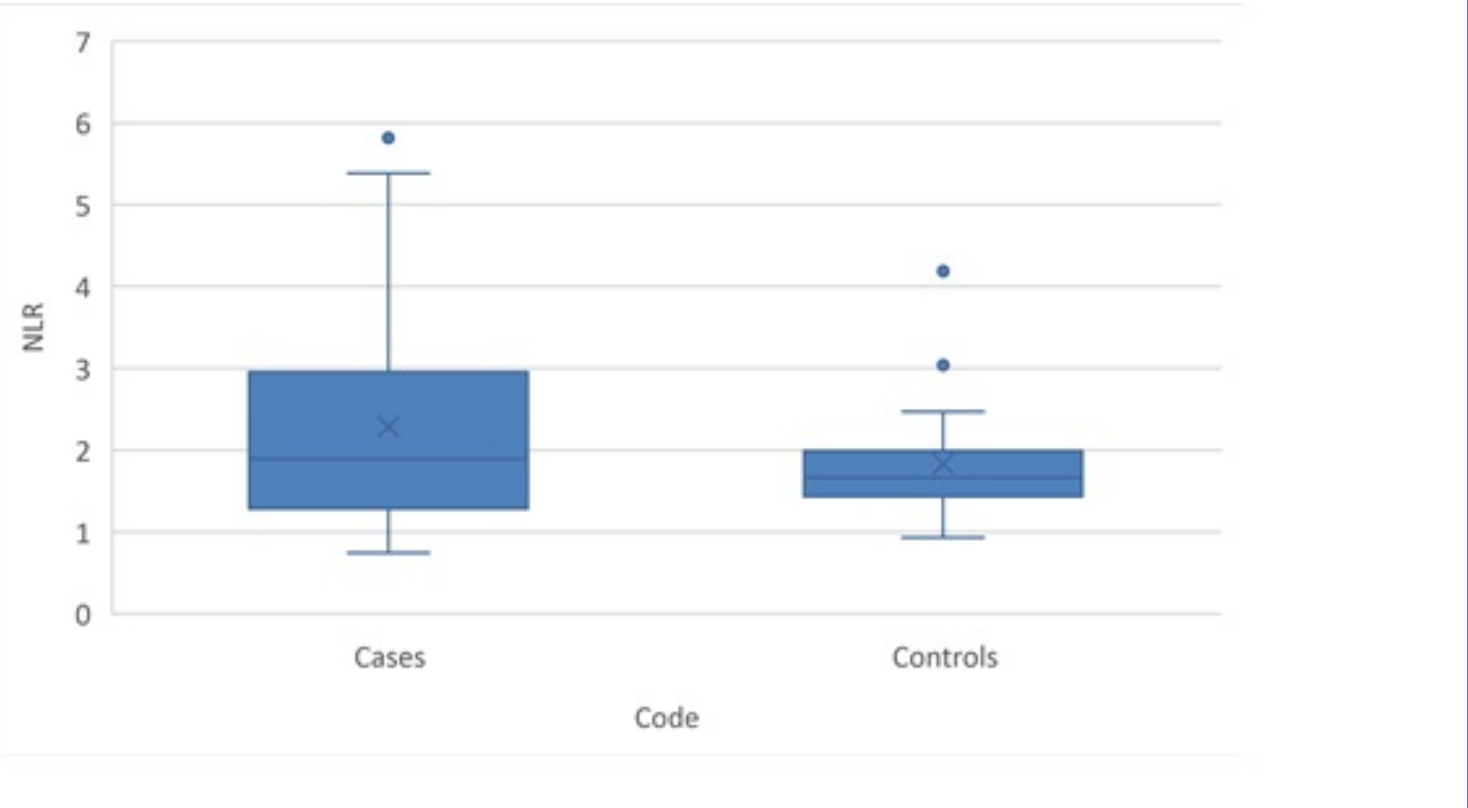

Fig. 3: Mean NLR in cases and controls.

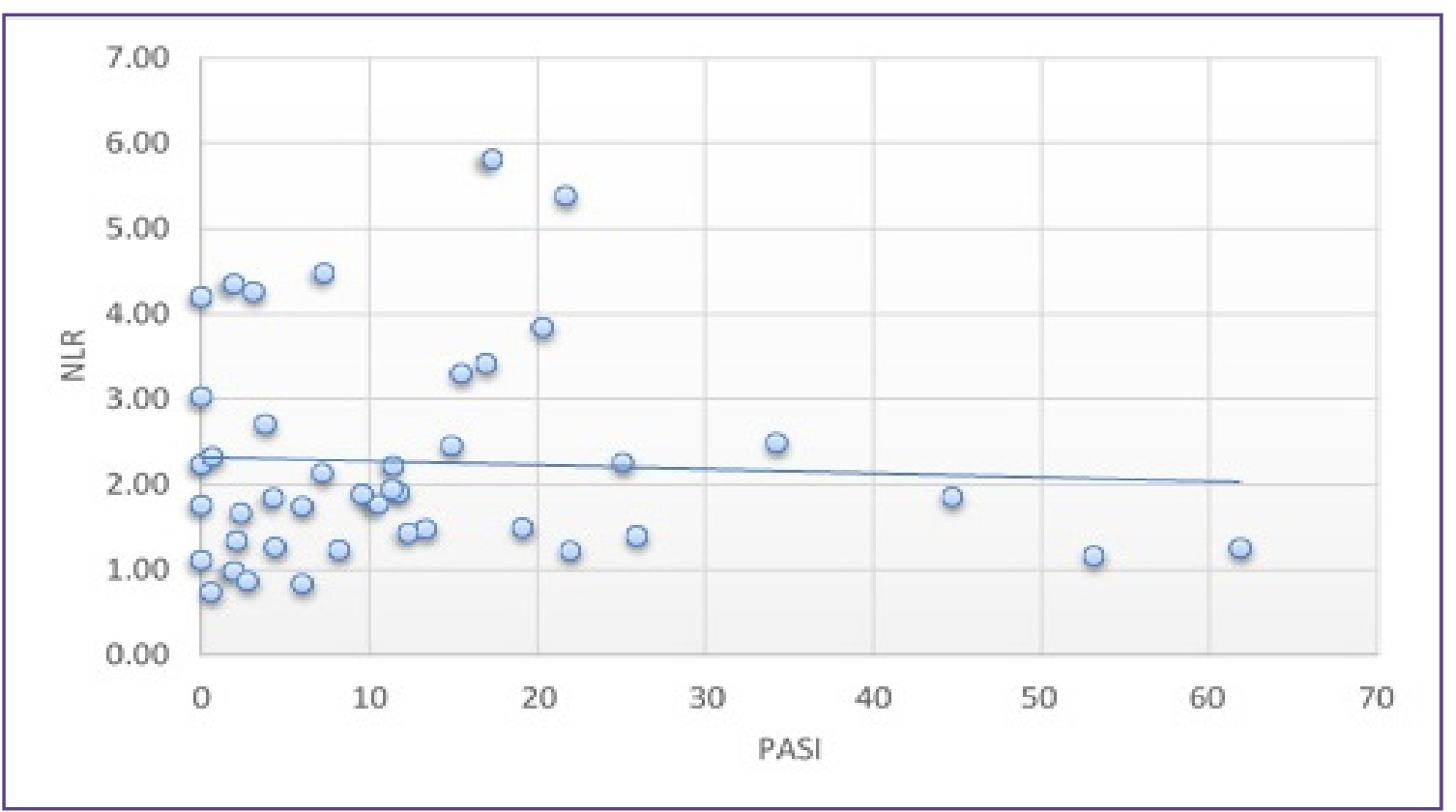

Fig. 4: Correlation between PASI and NLR $(r=0 .-39$, $p$ value $=0.811)$.

PASI score. Polat et al. ${ }^{[13]}$ reported higher PLR levels ( $\mathrm{p}$ value $=0.003)$ in psoriasis patients $(159.18 \pm 64.22)$ than the control group (125.01 \pm 38.14 ). Although, Kim et al. ${ }^{[4]}$ did not find increase in PLR in psoriasis patients than controls. However, similar to our study Polat et al. ${ }^{[13]} \mathrm{Kim}$ et al. ${ }^{[4]}$ and Asahina et al. ${ }^{[14]}$ found positive correlation between PASI and PLR.
Asahina et al. ${ }^{[14]}$ stated that both NLR and PLR levels were significantly lower in psoriasis vulagaris than psoriatic arthritis. They also showed a improvement of NLR and PLR values after medication. Yurtdas et al. ${ }^{[17]}$ correlated NLR and PLR with predictors of subclinical atherosclerosis like aortic velocity propagation and carotid intima-media thickness in psoriasis patients and showed a 
positive correlation with both NLR and PLR. This result should be seen in the light of platelet activation and its role in atherosclerosis and psoriasis.

PLR evaluation is rapid, low-cost and can be easily calculated. Therefore, it can be readily used in daily clinical practice without any extra efforts. The present study had some limitations due to its limited sample size. This study suggests the role of PLR as a biomarker in psoriasis, however, additional studies with larger sample size are certainly needed to validate its clinical use.

\section{Conclusion}

In conclusion we found that PLR was significantly elevated in psoriasis patients as compared to controls and it also correlates with the disease severity. PLR can be used as a biomarker to measure and monitor the disease activity in psoriasis patients at the time of diagnosis and follow up. This can also aid in preventing comorbidities in psoriasis patients.

\section{Reference}

1. Pariser DM, Bagel J, Gelfand JM, Korman NJ, Ritchlin CT, Strober BE, et al. National Psoriasis Foundation clinical consensus on disease severity. Arch Dermatol. 2007;143:239-42.

2. Dogra S, Mahajan R. Psoriasis: Epidemiology, clinical features, co-morbidities, and clinical scoring. Indian Dermatol Online J. 2016;7:471-80.

3. Villanova F, Di Meglio P, Nestle FO. Biomarkers in psoriasis and psoriatic arthritis. Ann Rheum Dis. 2013;72 Suppl 2:ii104-10.

4. Kim DS, Shin D, Lee MS, Kim HJ, Kim DY, Kim SM, et al. Assessments of neutrophil to lymphocyte ratio and platelet to lymphocyte ratio in Korean patients with psoriasis vulgaris and psoriatic arthritis. J Dermatol. 2016;43:305-10.

5. Paul C, Gourraud PA, Bronsard V, Prey S, Puzenat E, Aractingi $\mathrm{S}$, et al. Evidence-based recommendations to assess psoriasis severity: systematic literature review and expert opinion of a panel of dermatologists. J Eur Acad Dermatol Venereol. 2010;24 Suppl 2:2-9.

6. Uysal S, Yilmaz FM, Karatoprak K, Artuz F, Cumbul NU. The levels of serum pentraxin3, CRP, fetuin-A, and insulin in patients with psoriasis. Eur Rev Med Pharmacol Sci. 2014;18:3453-8.
7. Beygi S, Lajevardi V, Abedini R. C-reactive protein in psoriasis: a review of the literature. J Eur Acad Dermatol Venereol. 2014;28:700-11.

8. Azab B, Zaher M, Weiserbs KF, Torbey E, Lacossiere K, Gaddam S, et al. Usefulness of neutrophil to lymphocyte ratio in predicting short- and long-term mortality after non-ST-elevation myocardial infarction. Am J Cardiol. 2010;106:470-6.

9. Buyukkaya E, Karakas MF, Karakas E, Akcay AB, Tanboga IH, Kurt M, et al. Correlation of neutrophil to lymphocyte ratio with the presence and severity of metabolic syndrome. Clin Appl Thromb Hemost. 2014;20:159-63.

10. Imtiaz F, Shafique K, Mirza SS, Ayoob Z, Vart P, Rao S. Neutrophil lymphocyte ratio as a measure of systemic inflammation in prevalent chronic diseases in Asian population. Int Arch Med. 2012;5:2.

11. Fu H, Qin B, Hu Z, Ma N, Yang M, Wei T, et al. Neutrophiland platelet-to-lymphocyte ratios are correlated with disease activity in rheumatoid arthritis. Clin Lab. 2015;61:269-73.

12. Huang W, Huang J, Liu Q, Lin F, He Z, Zeng Z, et al. Neutrophil-lymphocyte ratio is a reliable predictive marker for early-stage diabetic nephropathy. Clin Endocrinol (Oxf). 2015;82:229-33.

13. Polat M, Bugdayci G, Kaya H, Oguzman H. Evaluation of neutrophil-to-lymphocyte ratio and platelet-to-lymphocyte ratio in Turkish patients with chronic plaque psoriasis. Acta Dermatovenerol Alp Pannonica Adriat. 2017;26:97-100.

14. Asahina A, Kubo N, Umezawa Y, Honda H, Yanaba K, Nakagawa H. Neutrophil-lymphocyte ratio, plateletlymphocyte ratio and mean platelet volume in Japanese patients with psoriasis and psoriatic arthritis: Response to therapy with biologics. J Dermatol. 2017;44:1112-21.

15. Raghavan V, Radha RKN, Rao RK, Kuberan A. A Correlative Study between Platelet Count, Mean Platelet Volume and Red Cell Distribution Width with the Disease Severity Index in Psoriasis Patients. J Clin Diagn Res. 2017;11:EC13-EC6.

16. Ataseven A, Bilgin AU, Kurtipek GS. The importance of neutrophil lymphocyte ratio in patients with psoriasis. Mater Sociomed. 2014;26:231-3.

17. Yurtdas M, Yaylali YT, Kaya Y, Ozdemir M, Ozkan I, Aladag N. Neutrophil-to-lymphocyte ratio may predict subclinical atherosclerosis in patients with psoriasis. Echocardiography. 2014;31:1095-104.

*Corresponding author:

Dr. Namrata Sarin, Head of the Department, Department of Pathology, North Delhi Municipal Corporation Medical College and Hindu Rao Hospital, Delhi, India 110007

Email: drnamrata50@gmail.com

Financial or other Competing Interests: None. 